\title{
From Research to Practice: Automated Negotiations with People
}

\author{
Raz $\operatorname{Lin}^{1}$ and Sarit Kraus ${ }^{1,2 \star}$ \\ 1 Department of Computer Science \\ Bar-Ilan University \\ Ramat-Gan, 52900 Israel \\ \{linraz, sarit\}@cs.biu.ac.il \\ 2 Institute for Advanced Computer Studies \\ University of Maryland \\ College Park, MD 20742 USA
}

\begin{abstract}
The development of proficient automated agents has flourished in recent years, yet making the agents interact with people has still received little attention. This is mainly due to the unpredictable nature of people and their negotiation behavior, though complexity and costs attached to experimentation with people, starting from the design and ending with the evaluation process, is also a factor. Even so, succeeding in designing proficient automated agents remains an important objective. In recent years we have invested much effort in facilitating the design and evaluation of automated agents interacting with people, making it more accessible to researchers. We have created two distinct environments for bargaining agents, as well as proposing a novel approach for evaluating agents. These are key factors for making automated agents become a reality rather than remain theoretical.
\end{abstract}

\section{Introduction}

Negotiation is the process in which interested parties exchange information in order to come to an agreement [3]. Thus, negotiation surrounds our every day life. This emphasizes the need for proficient automated negotiation agents which can interact with people in open environments. The introduction of open environments is important, as the automated agent needs to be able to interact with different types of people who have different characteristics, originate from different countries and cultures and so on. Automated negotiation agents capable of negotiating proficiently with people must therefore deal with the fact that people are diverse in their behavior and each individual might negotiate in a different manner.

Two main difficulties in the design of proficient automated agents that interact with people stem from the fact that people are involved. First, the design is not straightforward. It is not sufficient to design agents that follow economical paradigms and strive

\footnotetext{
* This research is based upon work supported in part by the U.S. Army Research Laboratory and the U.S. Army Research Office under grant number W911NF-08-1-0144 and under NSF grant 0705587 and by ERC grant \#267523.
} 
for the equilibrium, or to design agents that implement quantitative decision-making approaches and are expected utility maximizers, as results from social sciences suggest that people do not follow equilibrium strategies $[4,14]$. Human behavior is diverse and cannot be captured by a monolithic model. Humans tend to make mistakes, and they are affected by cognitive, social and cultural factors $[1,8]$. Moreover, when playing with people, the theoretical equilibrium strategy is not necessarily the optimal strategy [20]. In this respect, equilibrium based automated agents that play with people must incorporate heuristics to allow for "unknown" deviations in the behavior of the other party. In addition to the problems of the design of a proficient agent, the researcher also needs to evaluate the agent's interactions with people. The scientific evaluation of computational strategies for human-computer decision-making is a costly process, involving time, effort and personnel.

In recent years we have focused our efforts in making this process practical and reachable. In this paper we will describe two environments we have designed, the GENIUS environment [11], which is a bilateral negotiation environment, and the Colored Trails game environment [7], which is a game played on a $n \mathrm{x} m$ board of colored squares. These two environments allow the test-bedding of automated agents, as well as the matching of agents with other agents, people with people and people with automated agents. However, as we mentioned, designing agents is not suffice. Thus, we also showed how the evaluation process of the automated agents can be facilitated using peer-designed agents. Building on these, we hope to motivate more researchers in pursuing agents design and evaluation with people.

The remainder of this paper is organized as follows. In Section 2 we provide an overview of the GENIUS environment and describe an automated negotiator designed in GenIUS. We continue and describe the Colored-Trail environment and an automated agent designed in this environment in Section 3. In Section 4 we provide our novel approach for facilitating the evaluation process of automated agents. Finally, we provide a summary and discuss the results.

\section{The GeniUs Environment}

GeNIUs [11] is a General Environment for Negotiation with Intelligent multi-purpose Usage Simulation. With the constant introduction of new domains, e-commerce and other applications which require automated negotiations, generic automated negotiators encompass many benefits and advantages over agents that are designed for a specific domain. GENIUS both assists in the design of strategies for automated negotiators and also supports the evaluation process of the agent. Thus, we believe this tool is very useful for agent designers and can take a central part in the process of designing automated agents. While designing agents can be done in any agent-oriented software engineering methodology, GENIUS wraps this in an easy-to-use environment and allows the designers to focus on the development of strategies for negotiation in an open environment with multi-attribute utility functions.

GENIUS incorporates several mechanisms that aim to support the design of a general automated negotiator. The first mechanism is an analytical toolbox, which provides a variety of tools to analyze the performance of agents, the outcome of the negotiation 
and its dynamics. The second mechanism is a repository of domains and utility functions. As agents are built using the same infrastructure of GENIUS, GENIUS maintains a repository of automated agents and enables the evaluation and comparison of different strategies used by the automated agents. This is an important contribution as it allows researchers to empirically and objectively compare their agents with others in different domains and settings. This is an important contribution with respect to the validation of results reported by researchers with regard to their automated negotiators.

GENIUS supports a multi-issue negotiation setting in which participants engage in repeated negotiation rounds in which they can propose an agreement which consists of values for a subset or all of the issues in the negotiation. The negotiation protocol proceeds as follows: At each time period each participant can propose a possible agreement for some subset of the issues (see Figure 1), and the other participant can either accept the offer, reject it or opt out. The protocol is an extension of the classic alternating offers protocol of Osborne and Rubinstein ([16], p. 118-121) to support partial agreements as well as an opting-out option.

The negotiation terminates when one of the following holds: (a) the negotiators reach an agreement for all of the issues, (b) one of the participants opts out, thus forcing the termination of the negotiation with an default outcome, or (c) a predefined deadline $(d l)$ is reached, whereby, if a partial agreement is reached it is implemented or, if no agreement is reached, a status quo $(S Q)$ outcome is implemented. The utility for participants depends on the agreement and the time period in which it was reached.

Formally, let $I$ denote the set of issues in the negotiation, $O_{i}$ the finite set of values for each $i \in I$ and $O$ a finite set of values for all issues $\left(O_{1} \times O_{2} \times \ldots \times O_{|I|}\right)$. We allow partial agreements, $\perp \in O_{i}$, for each $i \in I$. Therefore an offer is denoted as a vector $\vec{o} \in O$.

The negotiation session is divided into time periods, Time $=\{0,1, \ldots, d l\}$. Each agent is assigned a time cost which influences its utility as time passes. In each period $t \in$ Time of the negotiation, if the negotiation has not terminated earlier, each agent can propose a possible agreement and the other agent can either accept the offer, reject it or opt out.

The negotiation problem also involves incomplete information about the preferences of the opponent. We assume that there is a finite set of agent types. These types are associated with different additive utility functions (e.g., one type might have a long term orientation regarding the final agreement, while the other type might have a more constrained orientation). Formally, we denote the possible types of agents as Types $=\{1, \ldots, k\}$. Given $l \in$ Types, $1 \leq l \leq k$, we refer to the utility of an agent of type $l$ as $u_{l}$, and $u_{l}:\{(O \cup\{S Q\} \cup\{O P T\}) \times$ Time $\} \rightarrow \mathbb{R}$. Each agent is given its exact utility function. The negotiators are aware of the set of possible types of the opponent. However, the exact utility function of the rival is private information.

In the next subsection we describe the KBAgent that was designed and evaluated using this system in two scenarios: an employer and an employee negotiating over terms of a job contract and a diplomatic negotiation process between two countries. Detailed score functions for both domains can be found in [12]. 


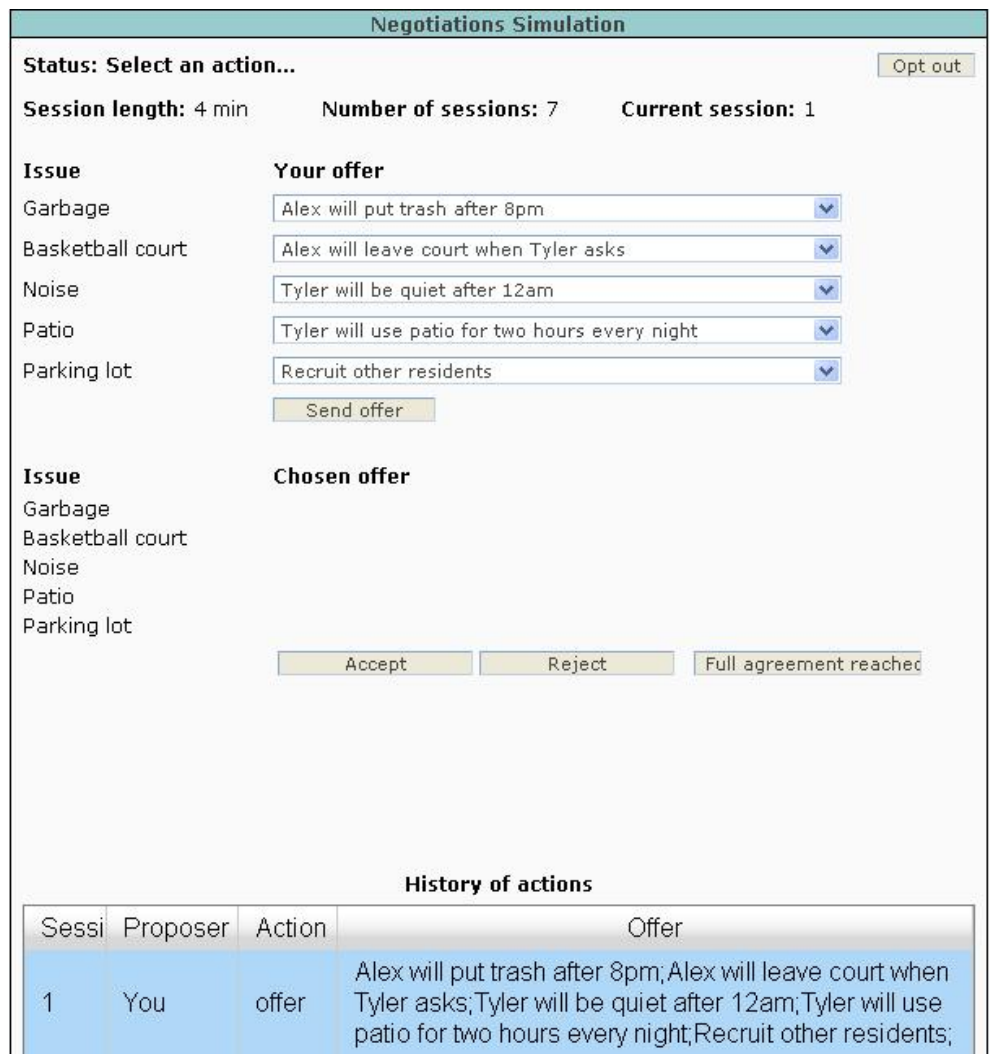

Fig. 1. Bilateral Negotiation: Generating offers screen.

\subsection{The KBAgent}

The KBAgent [17] is an automated negotiator that was proven to be proficient when negotiating with people. It incorporates machine learning techniques to allow it to generate a general model of the opponent and also follows qualitative decision-making, rather than quantitative. The KBAgent negotiates with each person only once, and uses a database of past negotiation sessions between specific agents (types of negotiators) to allow it to be more proficient in negotiations with agents of that specific type (in our experiments, we used a database of past negotiations of people to allow it to be matched with people). Based on a database with past negotiation sessions, the agent performs offline learning, which is based on the kernel-based density estimation ([21], Chapter 2). $K D E$ is a technique for estimating the probability functions based on samples, and can also be viewed as a smoothing technique based on samples. An advantage of this technique is that it does not assume a model of the other side, just like the data we expect to have in our database. From the database the agent estimates the probability of an offer to be accepted, the probability of it to be offered and the expected average utility for the other party. These probabilities are then used in its decision-making component, 
either when accepting an offer or to determine the agent's concession rate. Using the kernel-based density estimation the KBAgent is capable of using even small databases and does not have to rely on many past negotiation sessions. The KBAgent has been shown to negotiate proficiently with people [17]. In the remainder of this section we elaborate on the design of the KBAgent.

General Opponent Modeling Component This component is responsible for generating the general opponent modeling. The KBAgent uses the KDE method to estimate the probability of each offer to be proposed by the other party during a given turn. In addition, the database is used to calculate the probability of each offer to be accepted by the other side during any turn. We denote by $Q(\vec{o})$ the estimated probability of each offer $\vec{o} \in O$ to be accepted at any given time by the opposite party and by $P(\vec{o}, t)$ the probability that the opposite party would propose offer $\vec{o}$ during a given turn $t$. These probabilities will be used later in the decision-making process.

The database of past negotiations includes logs of past negotiation sessions between two sides in the specified domain. The negotiation sessions include all offers made by the two parties in each turn and whether or not the offers were accepted. The negotiation sessions may be collected from any population, not necessarily similar to the one which the KBAgent negotiates with.

The estimation of the probabilities is done separately for each possible agent type. If the negotiation sessions in the database are not labeled with the type of the agent, the type is elicited using a simple Bayes' classifier (similar to the one used for estimating the believed type of the other party during the negotiations). To estimate the probability of offers being proposed by the agent of a given type, we extract from the database all offers proposed by the agents of that type in any given turn $t, 1 \leq t \leq d l$. To apply the $K D E$ algorithm all offers have to be assigned a unique numerical value, thus we order the offers by the utility values of the other side and rank them, such that the offer with the highest possible utility value is ranked 1 . Then the $K D E$ is applied and the results also include a smooth probability value even for agreements that were not part of the samples in the database.

To estimate the probability that the other side will accept an offer during the negotiation we extract from the database all the offers ever accepted or proposed by at least one negotiator playing the role of the other party (under the assumption that if anyone had proposed an offer they would also accept it when proposed the same offer). We will refer to them as "acceptable offers". Note that we actually use the KDE method to obtain estimation on the utility of offers, not to learn any estimation on the offers themselves. For each offer during the negotiation that the KBAgent proposes, the acceptance probability is calculated by computing the ratio between (a) the number of the offers from the acceptable offer list that have a lower utility value for the other party than the utility value of the proposed offer, and (b) the total number of offers in the acceptable list of the other party.

We use the following example to demonstrate this. Assume that the database contains sessions in which people proposed offers with the following utilities: $\{400,380$, 300 , and 200$\}$. Also assume that they accepted an offer with a utility value of 280 . Thus, the acceptable offers list will contain the offers with the following utility values: $\{400$, 
$380,300,200$, and 280$\}$. Now assume that we would like to calculate the probability that the other party will accept an offer with a utility value of 290 . Since there are two agreements in the acceptable list with a utility value lower than 290 (280 and 200) the estimated probability of acceptance is $2 / 5=0.4$.

Finally, from the database we calculate the average expected utility of the other party. This is done by averaging the final utility scores of all agreements reached in the negotiation sessions (regardless of during which turn the agreements were reached). We denote this average as ExpectedOppAvg.

The Decision-Making Component The decision-making valuation component takes into account the agent's utility function, as well as the believed type of the opponent (note that the believed type of the opponent is also influenced by the offers proposed by the opponent). This data is used both for deciding whether to accept or reject an offer and for generating an offer. In our settings, although several offers can be proposed each time period, we restrict the agent to a single offer in each period. This is done due to the fact that our mechanism for generating offers only produces one distinct offer in a given time period. The opponent, on the other hand, is free to propose several offers, and the agent can respond to all the offers, which actually occurred in the experiments.

Generating Offers The KBAgent implements a concession-oriented strategy when generating offers. The process of deciding what offers to propose during each turn consists of three phases. First, a list of offers ranked by their QOValue is generated. The QOValue of an offer is an alternative to the Nash bargaining solution. In short, it tries, in a qualitative manner, to evaluate the offers based on the agent's utility and based on the likelihood of their acceptance by the other party (c.f. [12] for more details). The QOValue of an offer is calculated using the following Formula:

$$
\begin{aligned}
\operatorname{QOValue}(\vec{o}) & =\min \left\{\alpha_{o}, \beta_{o}\right\} \\
\text { where } \alpha_{o} & =\operatorname{rank}_{a}(\vec{o}) \cdot l u_{a}(\vec{o}) \\
\text { and } \beta_{o} & =\left[\operatorname{lu}_{a}(\vec{o})+\operatorname{lu}_{b}(\vec{o})\right] \cdot \operatorname{rank}_{b}(\vec{o})
\end{aligned}
$$

where $\operatorname{rank}(\cdot)$ is the ranking value of an offer, which is associated with each offer and a given utility function $u$. The rank number of an offer $\vec{o} \in O$ is calculated using the following formula:

$$
\operatorname{rank}(\vec{o})=\frac{\operatorname{order}(\vec{o}, O)}{|O|}
$$

where $\operatorname{order}(\cdot, \cdot)$ is the ordering function which places the offer $\vec{o}$ in an ordinal scale between 1 and $|O|$ according to its utility value compared to all other offers in $O$. $l u(\cdot)$ denotes the Luce number of an offer [13], which is a non-negative number that is associated with each offer. The Luce number of an offer $\vec{o} \in O$ is calculated using the following formula:

$$
l u(\vec{o})=\frac{u(\vec{o})}{\sum_{\vec{x} \in O} u(\vec{x})}
$$


After calculating the QOValue of each offer, the KBAgent orders all offers by their QOValue. The offer with the maximal QOValue is the first offer the KBAgent proposes. The second phase is to construct a new list which will be the one from which offers will be proposed. This new list is based on the sorted offers by their QOValue, in which the utility values for the KBAgent are above the value of the status quo. In addition, the KBAgent discards all offers with a lower QOValue that do not improve the other party's utility from offers with a higher QOValue. That is, if QOValue $\left(\overrightarrow{o_{i}}\right)<$ QOValue $\left(\overrightarrow{o_{j}}\right)$ and $u_{o p p}\left(\overrightarrow{o_{i}}\right)<u_{o p p}\left(\overrightarrow{o_{j}}\right)$ the offer $\overrightarrow{o_{i}}$ will be removed from the proposal list. The last phase is deciding which offer to propose each turn. As mentioned earlier, the first offer that is proposed is the one with the maximal QOValue. The other offers are picked from the ordered list based on the concession rate applied by the KBAgent and are chosen with a decreasing QOValue for the agent and an increasing utility value for the other party. Note that while we assume that the other party is not rational, we refer to a case where it does not maximize its expected utility, thus it should still prefer to accept offers with higher utility values than lower ones.

The concession rate is determined such that after passing $80 \%$ of the negotiation turns, the KBAgent will propose the offer closest to the ExpectedOppAvg, that is, the average expected utility value of the other party, as estimated from the database of past negotiations. In this way the KBAgent makes concessions while offering agreements which are still efficient for it. The concession rate basically determines the order in which offers in the offer list will be proposed. Thus, even if after $80 \%$ of the turns the other side rejects the offer, the KBAgent continues to propose offers from its list based on the concession rate.

Listing 1 describes the pseudo-code of the algorithm for generating the proposed offers and the concession rate.

We will demonstrate KBAgent's offer generation mechanism using the following example. Assume that in a given negotiation session there are 5 turns and 10 possible offers. Table 1 lists the possible offers sorted by their QOValue. Based on the utility value of each offer for the other party, it also lists the final ordered list of offers from which the KBAgent will propose offers to its counterpart. Now, assume that the average expected utility value of the opposite side, as estimated from the database, is 440 . Based on KBAgent's algorithm, after $80 \%$ of the turns, that is, after turn $4(5 \cdot 0.8=4)$, it should propose offer \#7, which is the first one with a higher utility value for the other party than the average expected utility determined from the database. This offer is the fifth offer in the ordered list of offers and thus the agent can now establish its concession rates and the offers to be proposed each turn. Since the KBAget aims to propose the fifth offer after turn 4 , the concession rate is determined as $5 / 4=1.25$. Thus, the agent should first propose the first offer in the list and then continue using the concession rate. That is, it should propose offers from its list in the the following order: $0,1.25,2.5,3.75,5,6.25$. The numbers are rounded down, so the KBAgent eventually proposes the offers ordered $0,1,2,3,5$ in its offer list, in that order. These offers are also marked in bold in Table 1.

Accepting Offers An important aspect of the agent's strategy is the decision of whether to accept or reject an offer. The KBAgent determines a time dependent threshold to 


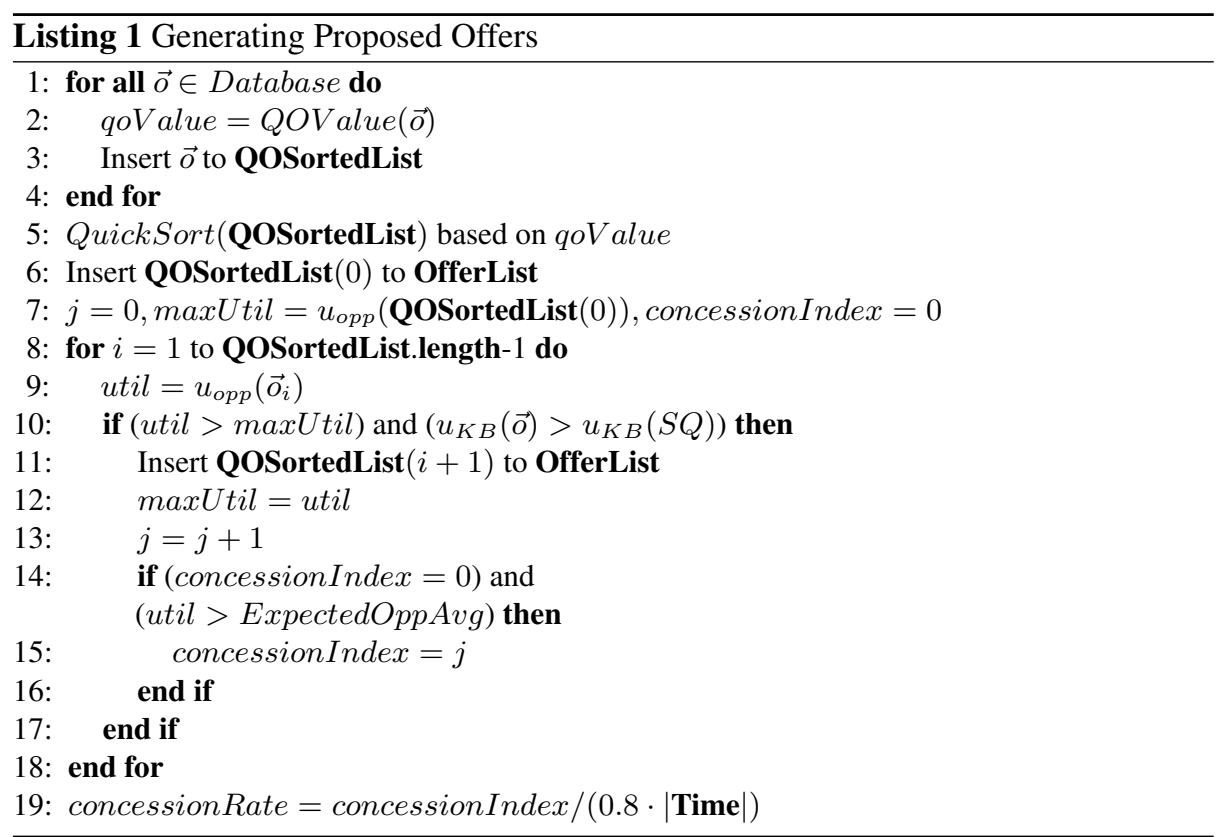

decide whether to accept or reject an offer. Obviously, a good threshold should be used and not an arbitrary one. In order to decide on the optimal threshold, the probabilities learned from the database of past negotiations are used.

Let $o_{K B}(t)$ be the offer proposed by the KBAgent at time $t$. The acceptance threshold for the KBAgent is calculated per every turn $t$ of the negotiation and denoted by $\alpha_{t}$. We want $\alpha_{t}$ to allow for maximization of the expected utility value of the agent for turn $t$. Thus, we first calculate the expected utility value of the KBAgent for each turn of the negotiation, denoted $E\left(t, \alpha_{t}\right)$. Since the negotiation terminated at turn $d l$ we can use backward induction to calculate this. The expected utility of the agent if the deadline is reached and no agreement has been made equals the utility of the status quo. Thus:

$$
E\left(d l, \alpha_{d l}\right)=u_{k b}(S Q)
$$

In the preceding turn, if KBAgent's offer was not accepted, it should accept any agreement with a utility value higher than $S Q$. Otherwise, the negotiation will terminate with a status quo outcome and a lower utility value for the agent. Thus, its expected utility depends on the probability that offers with a utility value higher than the status quo would be proposed by the other party. For all offers which are above the acceptance threshold of the KBAgent we sum their probability of being proposed by the counterpart multiplied by their utility value. For all other agreements, as the agent will reject them, we sum the probability of the counterpart proposing them multiplied by the value of the 


\begin{tabular}{|c|c|c|c|}
\hline \multicolumn{2}{|c|}{ Offer Idx $Q O V$ Vlue $(\vec{o})$} & $u_{\text {opp }}(\vec{o})$ & \multirow{2}{*}{$\begin{array}{l}\text { Ordered List of offers } \\
\mathbf{0}\end{array}$} \\
\hline 0 & 13.45 & 350 & \\
\hline 1 & 12.5 & 300 & - \\
\hline 2 & 12 & 400 & 1 \\
\hline 3 & 11.22 & 430 & 2 \\
\hline 4 & 10.3 & 350 & - \\
\hline 5 & 10 & 435 & 3 \\
\hline 6 & 9.87 & 470 & 4 \\
\hline 7 & 9.8 & 490 & 5 \\
\hline 8 & 9 & 410 & - \\
\hline 9 & 8.8 & 500 & 6 \\
\hline
\end{tabular}

Table 1. Example of deciding which offers will be proposed. Proposed offers are marked in bold.

status quo. Formally,

$$
\begin{array}{r}
E\left(d l-1, \alpha_{d l-1}\right)= \\
\sum_{u_{K B}(\vec{o}, d l-1) \geq \alpha_{d l-1}} P(\vec{o}, d l-1) u_{K B}(\vec{o}, d l-1)+ \\
\sum_{u_{K B}(\vec{o}, d l-1)<\alpha_{d l-1}} P(\vec{o}, d l-1) u_{K B}(S Q, d l-1)
\end{array}
$$

For every other turn, we calculate the expected utility of the KBAgent using a recursion. Basically, the expected utility of the agent at turn $i$ depends on whether or not it accepts the proposed offer:

- For all offers above the agent's acceptance threshold, the KBAgent sums the multiplication of their utility value with the probability that they will be proposed by its counterpart.

- For all offers below the agent's acceptance rate, the KBAgent calculates the probability that its offer will be accepted by its counterpart in the next turn or, if it is rejected, the agent's expected utility for the subsequent turn.

Formally,

$$
\begin{array}{r}
E\left(t, \alpha_{t}\right)=\sum_{u_{K B}(\vec{o}, t) \geq \alpha_{t}} P(\vec{o}, t) u_{K B}(\vec{o}, t)+ \\
\left(\sum_{u_{K B}(\vec{o}, t)<\alpha_{t}} P(\vec{o}, t)\right)\left\{Q\left(o_{K B}(t+1)\right) \cdot u_{K B}\left(o_{K B}(t+1), t+1\right)+\right. \\
\left.\left[1-Q\left(o_{K B}(t+1)\right)\right] E\left(t+1, \alpha_{t+1}\right)\right\}
\end{array}
$$


The last calculation needed is the extraction of $\alpha_{t}$ to determine the acceptance threshold for each turn. To this end, we use the derivative of Formula 6 by $\alpha_{t}{ }^{3}$, and as we would like to maximize the expected utility we find the $\alpha_{t}$ value when the derivative equals 0 . Thus we need to find the solution for the following equation:

$$
\begin{array}{r}
\alpha_{t}=\quad \begin{array}{r}
Q\left(o_{K B}(t+1)\right) u_{K B}\left(o_{K B}(t+1), t+1\right)+ \\
\left(1-Q\left(o_{K B}(t+1)\right)\right) E\left(t+1, \alpha_{t+1}\right)
\end{array}
\end{array}
$$

Based on Formulas 5, 6 and 7 we can backtrack and calculate the optimal acceptance threshold for each turn. Note that these calculations are done separately for each different believed agent type of the counterpart.

Identifying The Opponent's Type The KBAgent uses the same reasoning mechanism as suggested by Lin et al. [12] for identifying an opponent's type. This mechanism is based on the Bayesian updating rule that updates or revises beliefs in light of new evidence a posteriori ([9], Chapter 2). As incomplete information in the environment is modeled by the existence of several agent types in each time period, the agent consults the component in order to update its belief regarding the other party's type.

Recall that there are $k$ possible types of agents. At time $t=0$ the prior probability of each type is equal, that is, $\mathrm{P}\left(\right.$ type $\left._{t=0}^{i}\right)=\frac{1}{k}, \forall i \in$ Types. For each time period $t$ we calculate the a posteriori probability for each of the possible types, taking into account the history of the current negotiation. This is done incrementally after each offer is received or accepted. That is, the believed type is updated every time an offer is received or accepted, thus eventually it is based on the entire history thus far. Thereafter, this value is assigned to $\mathrm{P}\left(\right.$ type $\left._{t}\right)$. Using the calculated probabilities, the agent selects the type whose probability is the highest and proposes an offer as if it were the opponent's type. Formally, at each time period $t \in$ Time and for each type $\in$ Types and $\vec{o}_{t} \in O$ (the offer at time period $t$ ) we compute:

$$
\mathrm{P}\left(\text { type }^{i} \mid \vec{o}_{t}\right)=\frac{\mathrm{P}\left(\vec{o}_{t} \mid \text { type }^{i}\right) \mathrm{P}\left(\text { type }_{t}^{i}\right)}{\mathrm{P}\left(\vec{o}_{t}\right)}
$$

where $\mathrm{P}\left(\vec{o}_{t}\right)=\sum_{i=1}^{k} \mathrm{P}\left(\vec{o}_{t} \mid\right.$ type $\left.^{i}\right) \cdot \mathrm{P}\left(\right.$ type $\left._{t}^{i}\right)$.

Now we can deduce the believed type of the other party for each time period $t$, $B T(t)$, using the following equation:

$$
B T(t)=\underset{i \in \text { Types }}{\arg \max } \mathrm{P}\left(\operatorname{type}^{i} \mid \vec{o}_{t}\right), \quad \forall t \in \text { Time }
$$

\footnotetext{
${ }^{3}$ In order to perform this derivation we replace $\alpha_{t}$ with $u_{K B}\left(\beta_{t}, t\right)$ where $\beta_{t}$ is the threshold agreement, that is all offers with utility values higher than the utility if it were accepted. We then take derivative by $\beta_{t}$.
} 


\section{The Colored-Trail Environment}

The Colored Trails (CT) game [7] is a general negotiation test-bed that provides an analogy to task-settings in the real-world. ${ }^{4} \mathrm{CT}$ is played on a board of colored squares. Players are issued colored chips and are required to move from their initial square to a designated goal square. To move to an adjacent square, a player must turn in a chip of the same color as the square. Players must negotiate with each other to obtain chips needed to reach the goal square (see Figure 2). Each participant obtains a score at the end of the game that is computed as follows: $X$ points are given for reaching the goal square and $Y$ bonus points are given for each chip that each agent retains at the end of the game. If the player did not reach the goal, $Z$ penalty points are given for each square between the participant's final position and the goal square. Note that in this game, the performance of the agent does not depend on the outcome of the other player. Agreements are not enforceable, allowing players to promise chips but not transfer them. In addition, each player can see the entire game board. This environment is interesting for people to play as it is analogous to task settings and has a vivid representation of strategy space (as opposed to just a list of outcomes in the other environment).

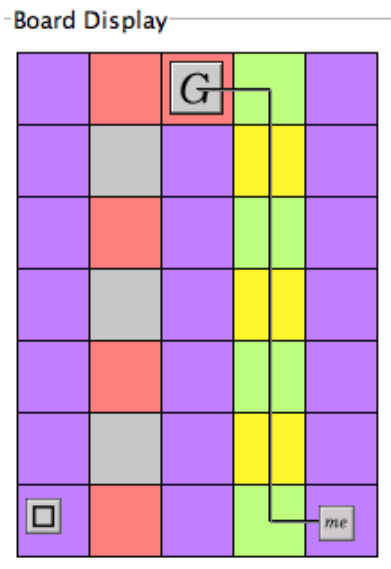

(a) Board Panel
Player Chips Display

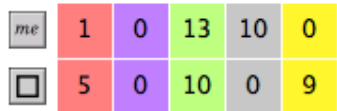

(b) Chip Display Panel (showing the chips in the possession of both participants)

Fig. 2. Snapshots of Colored Trails GUI

The simulation environment is adaptable such that different variations of the game can be set. The size of the board, the number and color of total chips and chips given to each player can be changed. In addition, the information accessible to each player can also be controlled (e.g., whether the player has full information of the others player's

\footnotetext{
${ }^{4}$ Colored Trails is Free Software and can be downloaded at http : / / www . eecs . harvard. edu/ai/ct
} 
chips). The automated agents can play both sides in the game, while the human counterpart accesses the game via a web address. The game itself is split into turns, where each turn is divided into a negotiation phase, a transfer phase and a movement phase. In the negotiation phase the players can request or promise to send chips. Then, in the transfer phase, the players choose the extent to which they meet their commitments by deciding the amount of chips to send. This might result in one agent sending the promised chips in return for the other agent's chips, while the other agent fails to deliver. In the movement phase, the players can choose to move to adjacent squares, given that they have the required colored chips. The game terminates when either side reaches the goal square or if any of the players has not moved in three consecutive turns. We continue to describe the PURB agent which was designed and evaluated in this environment using different variations of the game (board size, chips, dependency on the other player for reaching the goal square) $[5,6]$.

\subsection{The PURB Agent}

The PURB agent stands for Personality and Utility Rule Based agent. It combines a social utility function representing the behavioral traits of other participants with a rulebased mechanism using the utility function to make decisions in the negotiation process. This agent was designed to model and adapt its behavior to the individual traits exhibited by its negotiation partner by making trade-offs between acting selfishly to achieve short-term benefits and exhibiting helpful behavior towards maintaining long-term relationships. This is achieved by the agent dynamically changing its proclivity towards cooperation and reliability and also modeling the same traits in its opponent. A utility function is used to evaluate each possible action and proposal, and randomization is used to choose between different options [6]. As opposed to the KBagent described in Section 2.1, the PURB agent did not have access to previous negotiation data. It was therefore implemented with a set of rules which allow it to adapt its behavior based on current game play.

The agent negotiated with human subjects in the United States and Lebanon. Results showed that the agent was able to adopt a different negotiation strategy for each country. Its average performance across both countries was equal to that of people. However, the agent outperformed people in the United States because it learned to make offers that were likely to be accepted, and that were more beneficial to the agent than to people; the agent was outperformed by people in Lebanon, because it adopted a high reliability measure which allowed people to take advantage of it.

Utility Based Strategy The PURB agent modeled its negotiation partner in terms of two behavioral traits: The helpfulness trait of an agent described the willingness of negotiators to share resources with others. This was measured as the percentage of proposals in a game in which more chips were offered to the human than to the agent. The reliability trait of an agent described the degree to which negotiators kept their commitments. This was measured as the ratio between the number of chips transferred by a player after an agreement and the number of chips that were promised by the player. Together, these behavioral traits represented the cooperativeness measure of a negotiator. 
The social utility functions that the PURB agent employed are a weighted aggregate of features relating to its potential benefit from potential actions, and how these actions affected the way the human and the agent reasoned about each other's reliability and helpfulness over time.

For the remainder of this section we will use index $i$ to denote the PURB agent and index $j$ to denote the person.

For any state $s$ that encompasses a CT board, players' locations and chip settings, the social utility of the PURB agent, denoted $u_{i}$, was a function that was a weighted combination of the following features:

1. The expected future score for agent $i$. This score was computed using a heuristic that underestimated the benefit to the agent from a potential exchange in future rounds, given that the agent does not give any chips away. It was estimated as follows: Let $P_{i}(G \mid s, p)$ denote the probability that $i$ will get to the goal given that proposal $p$ is fulfilled. The expected future score was defined as

$$
\left(P_{i}(G \mid s, p) \cdot 100\right)+\left(\left(1-P_{i}(G \mid s, p) \cdot 100\right) \cdot 10 \cdot d\right)+s \cdot 5
$$

where 100 is the number of bonus points to get to the goal according to the CT scoring function; $d$ is the Manhattan distance of $i$ from the position on the board given that the exchange was fulfilled and the goal square; 10 is the number of penalty points for each square in the distance from the final position of PURB and the goal square; $s$ is the number of chips left in the player's possession after it advances to the goal using the shortest possible path, and 5 is the number of points awarded to the player for each chip left in its possession at the end of the game.

2. The expected future score for agent $j$ (computed in the same way as for $i$ ).

3 . The cooperativeness measure of agent $j$ (in terms of helpfulness and reliability). This feature represented $i$ 's beliefs of $j$ 's model of the reliability and helpfulness of $j$.

4. The cooperativeness measure of agent $i$ (in terms of helpfulness and reliability). This feature represented $i$ 's beliefs about how $j$ perceives its own measure of cooperativeness.

The weight of each aforementioned feature was established by a set of predetermined rules, and relied on the dependency relationships between participants as well as their cooperative measures.

Each time an agreement was reached and transfers were made in the game, $i$ updated the helpfulness and reliability measures of both agents (these values were aggregated over time using a discounting rate of 0.1 ).

Using this social utility allows the PURB agent to vary its strategy based on its estimate of the other participant's cooperativeness measure. For example, if the reliability of the other participant was high, this would increase the social utility of actions that favor the other participant.

The PURB agent also applied a set of rules that narrowed the search space of possible actions to be considered by the agent's utility function. These rules depended on aspects relating to the state of the game (e.g., the number of chips each agent had, whether a participant can independently reach the goal). At each step of the game, the 
agent used its social utility function to choose the best action out of the set of possible actions that were constrained by the rules. The rules were designed such that the $P U R B$ agent begins by acting reliably, and adapts over time to the individual measure of cooperativeness that is exhibited by its negotiation partner.

Generating Proposals The PURB agent generated a subset of possible offers based on its utility function. It non-deterministically chose any proposal out of the subset that provided a maximal benefit (within an epsilon interval) according to the agent's social utility function. A set of rules were applied to facilitate the agent's decision-making by reducing the set of possible proposals to consider when using its utility function.

Accepting Proposals The PURB agent accepted an offer if it was more advantageous to it than the offer it would make as a proposer in the same game state, or if accepting the offer was necessary to prevent the game from terminating. Formally, let $u_{i}\left(p_{j}\right.$, accept $\mid$ $s$ ) denote the utility for $i$ from an offer $p_{j}$ made by $j$ at state $s$. Let $p_{i}$ denote the offer that agent $i$ would make at state $s$. Generally, agent $i$ would accept an offer $p_{j}$ if $u_{i}\left(p_{j}\right.$, accept $\left.\mid s\right) \geq u_{i}\left(p_{i}\right.$, accept $\left.\mid s\right)$. In addition, $i$ would accept any proposal that prevented the game from ending, which occurs when the following conditions hold: (1) the chips in the possession of agent $i$ do not allow it to move on the board at state $s$; (2) the offer $p_{j}$ allows agent $i$ to move; and (3) if $i$ rejects the offer, the limit for dormant turns will be reached and the game would end.

Lastly, the degree to which $i$ accepted proposals from $j$ that made $j$ task-independent (i.e., they allowed $j$ to get to the goal) but where $i$ would remain task-dependent occurred only when $i$ was very certain that $j$ 's reliability was high. The degree of certainty of $i$ regarding the reliability of $j$ grew in proportion to the number of observations of $j$ 's behavior.

Transferring Chips These rules specify the extent to which the PURB agent fulfilled its agreements in the game. This behavior directly depended on its model of the other's cooperativeness. If the reliability of $j$ was low, it was likely that the other participants would not fulfill their agreement. Therefore $i$ did not send any of its promised chips. If the reliability of $j$ was high, then $i$ sent all of the promised chips. If the reliability of $j$ was medium, then the extent to to which $i$ was reliable was directly affected by the dependency relationships in the game:

1. If $j$ was task dependent, and the agreement will result in $j$ becoming task independent, then $i$ sent the largest set of chips such that $j$ remained task dependent.

2. If the exchange resulted in both agents becoming task independent, there was a high likelihood that $j$ will fulfil the agreement. In this case, $i$ sent all of the promised chips. Otherwise, $i$ sent half of the promised chips with a given probability, because it was not certain that $j$ would fulfil its agreements.

3. Lastly, if the exchange resulted in both agents being task dependent, then $i$ randomized between fulfilling its agreement and sending all of the promised chips, or sending a subset of the chips. Again, this is because $i$ was not certain that a participant with a medium measure of cooperativeness would meet its commitment. 
After the general description of the KBAgent (Section 2.1) and the PURB agent (Section 3.1), we describe in the next section how we employed a novel approach to evaluate the proficiency of these agents and assist in the process of their evaluation with people.

\section{Facilitating Agents' Evaluation}

Using people for the evaluation purposes is costly in terms of time, effort and money, making it a difficult task for researchers. The traditional view was that people cannot be replaced in the evaluation process because of their diverse behavior, which is affected by cognitive, social and cultural factors $[2,8]$.

In [10] we investigated the use of Peer Designed Agents (PDA) - computer agents developed by human subjects - as a tool for facilitating the evaluation process of automatic negotiators that were developed by researchers. We suggested a novel approach for evaluating the efficacy of automated agents by evaluating them with PDAs and not people. Thus, automated agents can be first evaluated and improved before moving on to experimentations with people.

This paradigm was inspired by the "strategy method" commonly used in behavioral economics, which is an experimental methodology that elicits from people a complete strategy specification. People state their actions for every possible situation that may arise in their interaction $[15,18,19]$. The assumption behind this method is that people are able to effectively encapsulate their own strategies if they are properly motivated, monetarily or otherwise.

In several different experiments we compared the performance of the KBAgent against people and against PDAs in the GENIUS environment, and the performance of the PURB agent with people and PDAs in the Colored-Trail game. The experiments included more than 300 human subjects and 50 PDAs developed by students.

Results show that PDAs can be used as a mechanism for evaluating automated negotiators that reflects on the behavior of people, as well as to fine-tune and improve the strategy of the automated negotiators. In particular, the KBAgent and the PURB agent were able to outperform people in our experiments in the same situations in which they were able to outperform PDAs. In addition, the results showed that the automated agents exhibited the same measure of generosity towards their negotiation partners. These patterns occurred for all types of domains, and for all types of automated negotiators, despite the fact that there were individual differences between the behavior of PDAs and people. These results provide an empirical proof that PDAs can be used to better determine the proficiency of an automated negotiator when matched with people, as well as to compare between the performance of different automated negotiators and their effects on human behavior. Thus, they can alleviate the evaluation process of automatic negotiators, and facilitate their design.

There are fundamental benefits to using PDAs instead of people. First, PDAs are accessible 24/7 and can be used whenever needed. In addition, PDAs are not biased and thus can be used several times to asses the automated agent's behavior. Thus they allow the agent designer to revise and change her agent with the ability to evaluate each 
design and compare it to previous designs. Lastly, it allows different automated agents to be matched on the same set of PDAs and obtain an objective evaluation of the results.

\section{Conclusions}

The importance of designing proficient automated negotiators to negotiate with people and evaluating them cannot be overstated. Yet, evaluating agents against people is a tiresome task, due to the cost and time required. In this paper we presented two infrastructures for designing agents and an overview of results of an extensive systematic experimentation. Our results revealed that playing well against peer designed agents can reflect on the proficiency of the automated negotiator when matched with people. Moreover, we showed that while PDAs result with different negotiation outcomes than people, there is a common behavioral pattern when they are both matched with automated agents.

The two infrastructures we presented - the GenIUS environment [11] and the Colored Trails game environment [7] - can be used for general purpose research on bargaining with people and automated agents, each with its distinct features. The GENIUS environment allows finite horizon bilateral negotiations between two people or agents. It allows the incorporation of any domain, with or without utility attached to it, and with or without a time discount factor. It also allows incorporation of mediator and statistical tools to assist during the negotiation. It basically follows an alternating offer protocol and can allow for unenforcable agreements. In addition it allows for complete or incomplete information, when incomplete information is expressed as uncertainty regarding the utility preferences of the opponent, and it is assumed that there is a finite set of different negotiator types. The second environment of the CT game is a general negotiation test-bed that provides an analogy to task-settings in the real world. Its vivid representation of strategy space (as compared to just a list of outcomes in the GENIUS environment) makes it more interesting for people to play. Like the GENIUS environment, automated agents can also play the game, and researchers can vary the different settings and complexity of the game (e.g., repeated vs. one-shot, availability of information, degree of dependency and communication protocol).

The two automated agents we described are also distinct from one another, not only by the environment in which each was used. The KBAgent is a domain independent agent, which implements several tactics and heuristics that are qualitative in nature. The main difference between it and the PURB agent is that it had access to data from previous interactions and used it in its decision-making component to increase its proficiency. It also used non-deterministic behavior via means of randomization. The PURB agent, on the other hand, is suited to be used in the CT game environment alone. Its non-deterministic behavior is based on rules which allow it to be adapted to emergent situations of the game, as it does not have access to past game data.

We have shown that it is indeed possible to develop automated agents that interact proficiently with people. The availability of generic infrastructures and the possibility of evaluating automated agents with peer designed agents to evaluate their performance with people should motivate more and more researchers to pursue the design of automated agents. Some of the drawbacks of designing automated negotiators that are 
capable of negotiating with people stems from the fact that their evaluation involves people and the cumbersome task of orchestrating the experiments with people. Perhaps simplifying this evaluation process could make the design of these agents more accessible and motivate researchers to design more automated agents that are specifically designed to negotiate proficiently with people. 


\section{Bibliography}

[1] M. H. Bazerman and M. A. Neale. Negotiator rationality and negotiator cognition: The interactive roles of prescriptive and descriptive research. In H. P. Young, editor, Negotiation Analysis, pages 109-130. The University of Michigan Press, 1992.

[2] C. F. Camerer. In Behavioral Game Theory. Experiments in Strategic Interaction, chapter 2. Princeton University Press, 2003.

[3] R. Davis and R. G. Smith. Negotiation as a metaphor for distributed problem solving. Artificial Intelligence, 20:63-109, 1983.

[4] I. Erev and A. Roth. Predicting how people play games: Reinforcement learning in experimental games with unique, mixed strategy equilibrium. American Economic Review, 88(4):848-881, 1998.

[5] Y. Gal, B. Grosz, S. Kraus, A. Pfefer, and S. Shieber. Agent decision-making in open mixed networks. AIJ, accepted, 2010.

[6] Y. Gal, S. Kraus, M. Gelfand, Y. Blumberg, and E. Salmon. An adaptive agent for negotiating with people in different cultures. ACM Transactions on Intelligent Systems and Technology (ACM TIST), accepted, 2010.

[7] B. Grosz, S. Kraus, S. Talman, and B. Stossel. The influence of social dependencies on decision-making: Initial investigations with a new game. In $A A M A S$, pages 782-789, 2004.

[8] D. A. Lax and J. K. Sebenius. Thinking coalitionally: party arithmetic, process opportunism, and strategic sequencing. In H. P. Young, editor, Negotiation Analysis, pages 153-193. The University of Michigan Press, 1992.

[9] T. Leonard and J. S. J. Hsu. Bayesian Methods - An Analysis for Statisticians and interdisciplinary Researchers. Cambridge University Press, Cambridge, UK, 1999.

[10] R. Lin, S. Kraus, Y. Oshrat, and Y. K. Gal. Facilitating the evaluation of automated negotiators using peer designed agents. In AAAI, pages 817-822, 2010.

[11] R. Lin, S. Kraus, D. Tykhonov, K. Hindriks, and C. M. Jonker. Supporting the design of general automated negotiators. In ACAN, 2009.

[12] R. Lin, S. Kraus, J. Wilkenfeld, and J. Barry. Negotiating with bounded rational agents in environments with incomplete information using an automated agent. AIJ, 172(6-7):823-851, 2008.

[13] R. D. Luce. Individual Choice Behavior: A Theoretical Analysis. John Wiley \& Sons, NY, 1959.

[14] R. D. McKelvey and T. R. Palfrey. An experimental study of the centipede game. Econometrica, 60(4):803-836, 1992.

[15] T. Offerman, J. Potters, and H. A. A. Verbon. Cooperation in an overlapping generations experiment. Games and Economic Behavior, 36(2):264-275, 2001.

[16] M. J. Osborne and A. Rubinstein. A Course In Game Theory. MIT Press, Cambridge MA, 1994.

[17] Y. Oshrat, R. Lin, and S. Kraus. Facing the challenge of human-agent negotiations via effective general opponent modeling. In $A A M A S$, pages 377-384, 2009. 
[18] R. Selten, K. Abbink, J. Buchta, and A. Sadrieh. How to play (3x3)-games: A strategy method experiment. Games and Economic Behavior, 45(1):19-37, 2003.

[19] R. Selten, M. Mitzkewitz, and G. R. Uhlich. Duopoly strategies programmed by experienced players. Econometrica, 65(3):517-556, 1997.

[20] A. Tversky and D. Kahneman. The framing of decisions and the psychology of choice. Science, 211:453-458, 1981.

[21] M. Wand and M. Jones. Kernel Smoothing. Chapman \& Hall, London, 1995. 